Behm, Britta; Rohstock, Anne

\title{
Loyalität. Zur verdeckten Regulierung von Denk-Bewegungen in wissenschaftlichen Feldern
}

van Ackeren, Isabell [Hrsg.]; Bremer, Helmut [Hrsg.]; Kessl, Fabian [Hrsg.]; Koller, Hans Christoph [Hrsg.]; Pfaff, Nicolle [Hrsg.]; Rotter, Caroline [Hrsg.]; Klein, Dominique [Hrsg.]; Salaschek, Ulrich [Hrsg.]: Bewegungen. Beiträge zum 26. Kongress der Deutschen Gesellschaft für Erziehungswissenschaft. Opladen; Berlin; Toronto : Verlag Barbara Budrich 2020, S. 51-70. - (Schriften der Deutschen Gesellschaft für Erziehungswissenschaft (DGfE))

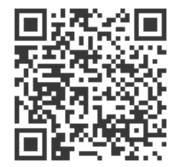

Quellenangabe/ Reference:

Behm, Britta; Rohstock, Anne: Loyalität. Zur verdeckten Regulierung von Denk-Bewegungen in wissenschaftlichen Feldern - In: van Ackeren, Isabell [Hrsg.]; Bremer, Helmut [Hrsg.]; Kessl, Fabian [Hrsg.]; Koller, Hans Christoph [Hrsg.]; Pfaff, Nicolle [Hrsg.]; Rotter, Caroline [Hrsg.]; Klein, Dominique [Hrsg.]; Salaschek, Ulrich [Hrsg.]: Bewegungen. Beiträge zum 26. Kongress der Deutschen Gesellschaft für Erziehungswissenschaft. Opladen; Berlin; Toronto : Verlag Barbara Budrich 2020, S. 51-70 - URN: urn:nbn:de:0111-pedocs-192330 - DOI: 10.25656/01:19233

https://nbn-resolving.org/urn:nbn:de:0111-pedocs-192330

https://doi.org/10.25656/01:19233

in Kooperation mit / in cooperation with:

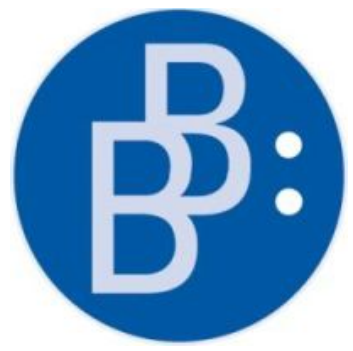

https://www.budrich.de

\section{Nutzungsbedingungen}

Dieses Dokument steht unter folgender Creative Commons-Lizenz: http://creativecommons.org/licenses/by-sa/4.0/deed.de - Sie dürfen das Werk bzw. den Inhalt vervielfältigen, verbreiten und öffentlich zugänglich machen sowie Abwandlungen und Bearbeitungen des Werkes bzw. Inhaltes anfertigen, solange sie den Namen des Autors/Rechteinhabers in der von inm festgelegten Weise nennen und die daraufhin neu entstandenen Werke bzw. Inhalte nur unter Verwendung von Lizenzbedingungen weitergeben, die mit denen dieses Lizenzvertrags identisch, vergleichbar oder kompatibel sind.

Mit der Verwendung dieses Dokuments erkennen Sie die Nutzungsbedingungen an.

\section{Terms of use}

This document is published under following Creative Commons-License: http://creativecommons.org/licenses/by-sa/4.0/deed.en - You may copy, distribute and transmit, adapt or exhibit the work or its contents in public and alter, transform, or change this work as long as you attribute the work in the manner specified by the author or licensor. New resulting works or contents must be distributed pursuant to this license or an identical or comparable license.

By using this particular document, you accept the above-stated conditions of use.

\section{Kontakt / Contact:}

peDOcs

DIPF | Leibniz-Institut für Bildungsforschung und Bildungsinformation

Informationszentrum (IZ) Bildung

E-Mail: pedocs@dipf.de

Internet: www.pedocs.de

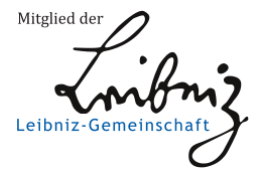




\section{Bewegungen}

Beiträge zum 26. Kongress der Deutschen Gesellschaft für Erziehungswissenschaft

Isabell van Ackeren, Helmut Bremer, Fabian Kessl, Hans Christoph Koller, Nicolle Pfaff, Caroline Rotter, Dominique Klein, Ulrich Salaschek (Hrsg.)

Schriften der Deutschen Gesellschaft für Erziehungswissenschaft

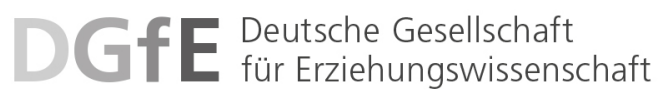


Isabell van Ackeren

Helmut Bremer

Fabian Kessl

Hans Christoph Koller

Nicolle Pfaff

Caroline Rotter

Dominique Klein

Ulrich Salaschek (Hrsg.)

\section{Bewegungen}

Beiträge zum 26. Kongress der Deutschen Gesellschaft für Erziehungswissenschaft

Verlag Barbara Budrich

Opladen • Berlin • Toronto 2020 
Bibliografische Information der Deutschen Nationalbibliothek

Die Deutsche Nationalbibliothek verzeichnet diese Publikation in der Deutschen Nationalbibliografie; detaillierte bibliografische Daten sind im Internet über

http://dnb.d-nb.de abrufbar.

(C) 2020 Dieses Werk ist bei der Verlag Barbara Budrich GmbH erschienen und steht unter der Creative Commons Lizenz Attribution-ShareAlike 4.0 International (CC BY-SA 4.0):

https://creativecommons.org/licenses/by-sa/4.0/.

Diese Lizenz erlaubt die Verbreitung, Speicherung, Vervielfältigung und Bearbeitung bei

Verwendung der gleichen CC-BY-SA 4.0-Lizenz und unter Angabe der UrheberInnen, Rechte, Änderungen und verwendeten Lizenz.

www.budrich.de

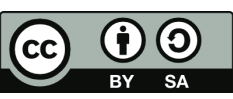

Dieses Buch steht im Open-Access-Bereich der Verlagsseite zum kostenlosen Download bereit (https://doi.org/10.3224/84742385).

Eine kostenpflichtige Druckversion (Print on Demand) kann über den Verlag bezogen werden. Die Seitenzahlen in der Druck- und Onlineversion sind identisch.

$$
\begin{array}{ll}
\text { ISBN } & 978-3-8474-2385-0 \text { (Paperback) } \\
\text { eISBN } & 978-3-8474-1553-4 \text { (PDF) } \\
\text { DOI } & 10.3224 / 84742385
\end{array}
$$

Druck: paper \& tinta, Warschau

Printed in Europe auf säurefreiem und alterungsbeständigem Papier

Umschlaggestaltung: Bettina Lehfeldt, Kleinmachnow - www.lehfeldtgraphic.de

Redaktion und Satz: Fabian Auer, Wuppertal

Typographisches Lektorat: Anja Borkam, Jena - kontakt@lektorat-borkam.de 


\section{Inhalt}

Hans-Christoph Koller

Vorwort

Fabian Kessl, Nicolle Pfaff, Isabell van Ackeren, Helmut Bremer,

Hans-Christoph Koller, Carolin Rotter, Dominique Klein, Ulrich Salaschek

Einleitung .

Käte Meyer-Drawe

Bewegungen: Viele Gemeinsamkeiten und noch mehr Unterschiede

\section{Teil I Denkbewegungen}

[Koordination: Fabian Kessl]

Christiane Thompson

„Science, not silence“. Die Öffentlichkeit der Universität an ihren Grenzen

Barbara Rendtorff, Eva Breitenbach

Frauenbewegungen, Bildung und Erziehung - Erträge und Problematiken

Britta Behm, Anne Rohstock

Loyalität. Zur verdeckten Regulierung von Denk-Bewegungen in wissenschaftlichen

Feldern. Eine Sondierung am Beispiel der Geschichte westdeutscher Bildungsforscher .... 51

Fabian Kessl

Bewegungen an den Grenzen des Disziplinären: das Beispiel von Sozialpädagogik und Sozialer Arbeit. 71

Susann Fegter, Karen Geipel, Anna Hontschik, Bettina Kleiner, Daniela Rothe, Kim-Patrick Sabla, Maxine Saborowski

Äußerungen von Sprecher*innen in einer Gruppendiskussion. Überlegungen und Analysen aus unterschiedlichen diskurs- und subjektivierungstheoretischen Perspektiven 83

Teil II Migrationsbewegungen

[Koordination: Nicolle Pfaff]

Paul Mecheril

Gibt es ein transnationales Selbstbestimmungsrecht? Bewegungsethische

Erkundungen 101 


\section{Thomas Geier}

Integration ohne Ende. Kritische Stichworte zum monothematischen Habitus der

Migrationsdebatte in Deutschland

Marcus Emmerich, Ulrike Hormel, Judith Jording, Mona Massumi

Migrationsgesellschaft im Wandel - Bildungssystem im Stillstand?

Patricia Stošić, Benjamin Rensch

„Ja, (...) wären Sie denn nicht bereit, den Lehrerberuf aufzugeben?“

Bildungsbiographische Positionierungen muslimischer Lehramtsstudentinnen im

Spannungsfeld von Pluralismusdiskurs und Diskriminierung

Arnd-Michael Nohl

Politische Erziehung. Ein blinder Fleck der Diskussion zur politischen Bildung 161

Teil III Gesellschaftliche Entwicklungen und pädagogisches Tun

[Koordination: Fabian Kessl]

Johannes Bellmann, Dirk Braun, Martina Diedrich, Katharina Maag Merki, Marcelo Parreira do Amaral, Kate Maleike

„Wer steuert die Bildung - Wer steuert die Schule?“

Ein öffentliches Podiumsgespräch zur Eröffnung des 26. Kongresses der Deutschen

Gesellschaft für Erziehungswissenschaft

Anke Wischmann, Andrea Liesner

Neu zugewanderte Jugendliche zwischen engagierter pädagogischer Hilfe,

politischen Interessen und wirtschaftlichem Kalkül

Alisha M.B. Heinemann

Learning from below - Wissen in Bewegung. $\mathrm{Zu}$ den Möglichkeiten solidarischer

Bildungsarbeit durch den 'Funds of Knowledge-Approach'

Sebastian Wachs, Wilfried Schubarth, Ludwig Bilz

Hate Speech als Schulproblem? Erziehungswissenschaftliche Perspektiven auf ein aktuelles Phänomen

Teil IV Professionalisierung in der Lehrer*innenbildung

[Koordination: Carolin Rotter]

Alexander Gröschner

Praxisbezogene Lerngelegenheiten am Beispiel lernwirksamer

Unterrichtskommunikation. „Bewegungen“ in der Aus-, Fort- und Weiterbildung

von Lehrpersonen

Julia Košinár, Anna Laros

Orientierungsrahmen im Wandel? Berufsbiographische Verläufe zwischen Studium und Berufseinstieg 
Matthias Proske, Petra Herzmann, Markus Hoffmann

Spielfilme über Lehrer/innen als Medium der kasuistischen Lehrerbildung

Kristina Geiger, Petra Strehmel

Personalentwicklung in Kindertageseinrichtungen: Maßnahmen und Strategien

von Trägern und Einrichtungen. Ergebnisse zweier empirischer Studien 283

Christina Buschle, Tina Friederich

Weiterbildung als Motor für den Erhalt von Professionalität?

Weiterbildungsmöglichkeiten für das Kita-Personal

Nikolaus Meyer, Dieter Nittel, Julia Schütz

Was haben Erzieher*innen und Professor*innen gemeinsam? Komparative

Perspektiven auf zwei stark kontrastierende pädagogische Berufsgruppen. 309

\section{Teil V Digitalisierung}

[Koordination: Isabell van Ackeren]

Manuela Pietraß

Bildung in Bewegung. Das neue Lernpotenzial digitaler Medien

Mandy Schiefner-Rohs, Sandra Hofhues, Sandra Aßmann, Taiga Brahm

Studieren im digitalen Zeitalter. Methodologische Fragen und ein empirischer Zugriff... 337

Birgit Eickelmann, Kerstin Drossel

Lehrer*innenbildung und Digitalisierung - Konzepte und Entwicklungsperspektiven .... 349

Matthias Rohs, Manuela Pietraß, Bernhard Schmidt-Hertha

Weiterbildung und Digitalisierung. Einstellungen, Herausforderungen und Potenziale ... 363

Rudolf Kammerl, Jane Müller, Claudia Lampert, Marcel Rechlitz, Katrin Potzel

Kommunikative Figurationen - ein theoretisches Konzept zur Beschreibung von

Sozialisationsprozessen und deren Wandel in mediatisierten Gesellschaften? 377

\section{Teil VI Steuerung}

[Koordination: Dominique Klein]

Michael Schemmann

„Und sie bewegt sich doch“ - Neue Steuerung und Governance in der öffentlichen

Weiterbildung.

Katharina Maag Merki

Das Educational Governance-System im Dienste der Schulentwicklung. Oder:

Wie kann Steuerung die Weiterentwicklung von Schulen unterstützen? 405 


\section{Sigrid Hartong, Annina Förschler}

Dateninfrastrukturen als zunehmend machtvolle Komponente von Educational Governance. Eine Studie zur Implementierung und Transformation staatlicher Bildungsmonitoringsysteme in Deutschland und den USA....

Tobias Feldhoff, Sabine Reh, Eckhard Klieme, Monika Mattes, Sebastian Wurster,

Brigitte Steinert, Julia Dohrmann, Christine Schmid

Schulkulturen im Wandel - Potentiale und erste Erkenntnisse zur Untersuchung von

Schulkulturen im Wandel

Felix Berth, Mariana Grgic

Wie kam die Bildung in die Krippe? Frühe Kindertagesbetreuung im Spiegel von

Wissenschaften, Recht und individuellen Einstellungen in Westdeutschland seit den 1960er-Jahren

Teil VII Körper - Leib - Bewegung

[Koordination: Fabian Kessl \& Ulrich Salaschek]

André Gogoll, Erin Gerlach

Bewegung, Sport und Lernen - zwischen pädagogischem Wunsch und empirischer

Wirklichkeit.

Maike Groen, Hannah Jäkel, Angela Tillmann, Ivo Züchner

E-Sport - Ambivalenzen und Herausforderungen eines globalen, jugendkulturellen

Phänomens.

Nino Ferrin, Benjamin Klages

Zur Kultivierung utopischer Bewegungen. Markierungen des Nicht-Verfügbaren in der Academia.

Juliane Noack Napoles

Identität als Stillstand. Ein metaphernanalytischer Blick auf eine Nicht-Bewegung.....

\section{Teil VIII Diversity / Inklusion}

[Koordination: Nicolle Pfaff]

Barbara Asbrand, Julia Gasterstädt, Anja Hackbarth, Matthias Martens

Was bewegt Inklusion? Theoretische und empirische Analysen zu

Spannungsverhältnissen einer inklusiven Schule

\section{Nina Thieme}

Zur Charakteristik der Gesellschaft, an der im Zuge von Inklusion Teilhabe ermöglicht werden soll. Vergewisserungen und Reflexionen zu möglichen Implikationen 
Bernhard Rauh, Yvonne Brandl, Michael Wininger, David Zimmermann

Inklusionspädagogik - eine halbierte Bewegung? Psychoanalytische Perspektiven

auf ein erziehungs-wissenschaftliches Paradigma

Christian Stöger

„Aber Österreich darf nicht zurückbleiben!“ Zur Wiener Hilfsschulentwicklung um 1900 . 555

Anke Karber, Gülsen Sevdiren, Kerstin Heberle, Anne Schröter, Janieta Bartz,

Tatiana Zimenkova

Hochschuldidaktische Betrachtungen differenzreflexiver Lehrer*innenbildung.

Tanja Sturm, Benjamin Wagener, Monika Wagner-Willi

Inklusion und Exklusion im Fachunterricht. Ambivalente Relationen in Schulformen

der Sekundarstufe 1

Teil IX Soziale - pädagogische Bewegungen

[Koordination: Helmut Bremer \& Jana Trumann]

Patrick Bühler

Böse Mütter im Summer of Love. Antipädagogik und Psychotherapie in den

Siebziger-Jahren

Marcel Eulenbach, Thorsten Fuchs, Yagmur Mengilli, Andreas Walther,

Christine Wiezorek

„Ich möchte Teil einer Jugendbewegung sein“"? - Jugendkultur, Protest, Partizipation.... 613

Sabrina Schenk, Britta Hoffarth, Ralf Mayer

Populismus, Protest - und politische Bildung. Soziale Bewegung(en) in

Spannungsfeldern von Affektivität, Rationalität und Praktiken der Kritik

im öffentlichen Raum

Aziz Choudry

Activist learning and knowledge production.

Autorinnen und Autoren 653 


\title{
Loyalität. Zur verdeckten Regulierung von Denk-Bewegungen in wissenschaftlichen Feldern
}

\author{
Eine Sondierung am Beispiel der Geschichte \\ westdeutscher Bildungsforscher
}

\section{Loyalität als analytische Kategorie Historischer Bildungsforschung: Ausgang und Ansatzpunkte}

Die Regulierung von Denk-Bewegungen in wissenschaftlichen Feldern wird in der Wissenschaftsforschung üblicherweise als eine der zentralen Aufgaben wissenschaftlicher Gemeinschaften analysiert (vgl. z.B. Gläser 2006). Die hervorragende Bedeutung der Peers ist dabei ebenso analysiert und anerkannt, wie Macht- und Herrschaftsgefälle, etwa in Bezug auf sogenannte Gatekeeper, kritisch betrachtet werden können, besonders aus handlungs- bzw. feldtheoretischer Perspektive (grundlegend Bourdieu 1979/1987) und von feministischen Positionen aus (weiterführend Kunadt et al. 2014). Allerdings sind konkrete Praktiken der Regulierung auf der Mikroebene in der deutschen wie der internationalen Historischen Bildungsforschung, etwa im Hinblick auf die eigene zeithistorische Fachgeschichte, bislang eher selten thematisiert. Im vorliegenden Beitrag sollen diese Praktiken im Mittelpunkt stehen. Sofern sie bislang in den Blick gelangten, wurden sie zumeist entlang solcher Prozesse rekonstruiert, die mit formalisierten, im fachlichen Raum be- und anerkannten Leistungsindikatoren verbunden sind. Eine dieser Ausnahmen ist zum Beispiel die Untersuchung pädagogischer Berufungsverfahren an Universitäten der französischen Besatzungszone nach 1945 (Kersting 2008).

Auch eine solche Vorgehensweise lässt nach Wahrnehmung der Autorinnen des vorliegenden Beitrags immer noch substantielle Aspekte ausgeblendet. Gemeint sind Erwartungen an das alltägliche Verhaltens- und Gefühlsrepertoire der Wissenschaftler*in und entsprechende Praktiken jenseits der von ihr jeweils hervorgebrachten ,messbaren " wissenschaftlichen Produkte, die im wissenschaftlichen Alltag zwar durchgängig präsent sind, aber in der Regel verdeckt bleiben. Gespeist sowohl durch eigene Beobachtungen im Wissenschaftsall- 
tag als auch durch die Auseinandersetzung mit der Geschichte der westdeutschen Bildungsforschung hatten wir parallel die Vermutung entwickelt, dass hierbei der „Loyalität“ eine herausragende Rolle zukommt. Insofern interessiert uns Loyalität im Folgenden als Aspekt der Regulierung von Denk-Bewegungen im (erziehungs-)wissenschaftlichen Feld. Diese untersuchen wir - notwendig kursorisch - an zwei bewusst unterschiedlich gewählten Beispielen aus dem Umfeld der Geschichte der westdeutschen Bildungsforschung in ihrer ersten Phase, zwischen den 1950er und den späten 1970er Jahren (dazu Behm 2017). Dabei wird es auch darum gehen, Loyalität als historisch-analytische Kategorie zu sondieren beziehungsweise Möglichkeiten der Erforschung dieser Art von Phänomenen über die von uns jeweils different gewählten Zugänge zu dieser Thematik zur Diskussion zu stellen, ohne bereits eine Synthese vorzuschlagen.

In Bezug auf den Begriff der Loyalität setzen wir ein wissenschaftlich informiertes Alltagsverständnis voraus und verzichten an dieser Stelle auf eine exaktere Bestimmung. Das hängt neben der Spezifik des historiographischen Ansatzes, der phasenweise gezielt induktiv ausgerichtet ist, mit den Kennzeichen dieses Forschungsgebiets zusammen. So besitzt zwar jedes Mitglied einer wissenschaftlichen Fachgemeinschaft vermutlich ein profundes Alltagswissen zum Thema, wissens- und wissenschaftsgeschichtlich betrachtet aber wissen wir über die Funktionalität von Loyalitätsentwürfen und -beziehungen im wissenschaftlichen Feld noch wenig: Zum einen ist Loyalität in der Historischen Bildungsforschung bis dato noch nicht bearbeitet und wird international in der historischen Forschung erst seit einigen Jahren, vor allem im Zuge des Aufschwungs der Gefühlsgeschichte als Thema gewählt, die im deutschsprachigen Raum seit etwa 2005 auf der Agenda steht (Frevert 2009: 184, Osterkamp/Schulze Wessel 2017b: 2ff.). Zum anderen weisen bereits die wenigen vorliegenden Studien zur Geschichte der Loyalität ein heterogenes Verständnis des Begriffs auf. Loyalität wird nicht nur in zeitlich und institutionell unterschiedlichen Kontexten untersucht, etwa in der Geschichte von Organisationen, Nationalstaaten oder Empires (vgl. z.B. die Beiträge in Osterkamp/Schulze Wessel 2017a). Darüber hinaus wird auch der Terminus als analytische Kategorie in Referenz auf diverse Ansätze, vor allem aus der Soziologie, der Psychologie und der (Sozial-)Philosophie, unterschiedlich konzipiert (weiterführend z.B. Connor 2007, Iser 2008). Eine ,allgemeine Theorie der Loyalität“" ist noch nicht geschrieben (Osterkamp/Schulze Wessel 2016: 554). Im Fluss bleibt damit auch die grundlegende kategoriale Frage, ob Loyalität ,eigentlich“ ein Gefühl sei (und wie dieses theoretisch zu fassen wäre) oder beispielsweise eher eine Werthaltung und Praxis im Sinne Aristotelischer Tugend. Zudem mangelt es an Trennschärfe gegenüber ähnlichen Begriffen, besonders gegenüber dem der Treue (so z.B. bei Iser 2008: Sp. 731), nachgelagert etwa gegenüber dem des Gehorsams oder der Solidarität. Entsprechend verzichten auch die Historiker*innen Jana Osterkamp und Martin Schulze Wessel in ihrem knappen Forschungsüberblick auf eine Definition und sammeln zunächst „important analytical conceptual elements of loyalty“, nämlich „duration, mutuality, sequentiality and communication” (Osterkamp/Schulze Wessel 2017b: 3). Zu ergänzen ist, dass in historischen Studien zum Thema übereinstimmend Bezug auf relativ dauerhaft wirkende Prozesse der Konstituierung respektive der Sicherung von Mitgliedschaft, Zugehörigkeit und/oder von Identität genommen wird.

Damit zu unseren Beispielen, die mit Friedrich Edding (1909-2002) und Hellmut Becker (1913-1993) zwei der maßgeblichen Akteure der ersten Phase westdeutscher Bildungsforschung in den Blick nehmen. Beide Beiträge gehen Funktionen von Loyalität für die Regulierung von Wissenschaftsfeldern nach, setzen aber unterschiedlich an: Britta Behm geht es in einem biographischen Zugriff auf Edding um Loyalität beim Wechsel zwischen wissenschaftlichen Organisationen und in Eddings Beziehung zu Becker, der zwischen 1963 und 
1981 Leiter des Max-Planck-Instituts für Bildungsforschung (MPIB) war (Part 2). Anne Rohstock untersucht Beckers Biografie in der NS-Zeit und seine Loyalität gegenüber Elitezirkeln und Intellektuellen des NS-Regimes nach 1945. Am Beispiel Beckers zeigt sie, welche Wirkungen Loyalität in wissenschaftlichen und (wissenschafts-)politischen Netzwerken der Bundesrepublik hatte (Part 3).

\section{Friedrich Edding - Loyalität gegenüber Organisationen und dem Leiter des Max-Planck-Instituts für Bildungsforschung (MPIB) ${ }^{1}$}

Bei der biographischen Beschäftigung mit dem Bildungsökonomen Friedrich Edding wird besonders in seiner Autobiographie erkennbar, dass für ihn das Thema der Loyalität zeitlebens relevant und oft problematisch war, auch wenn er dies nicht expressis verbis adressierte (vgl. Edding 1989/überarb. 2000). ${ }^{2}$ Das ist an dieser Stelle nicht auszuführen, vielmehr werden im Folgenden zwei Beispiele skizziert, um sich Anlässen, Formen und Wirkungen von Loyalität im Feld der Bildungsforschung historiographisch zu nähern. Ausgewählt wurden zwei typische Situationen des Wissenschaftsalltags: zum Ersten der Wechsel Eddings von der Hochschule für Internationale Pädagogische Forschung (HIPF) in Frankfurt am Main an das Berliner MPIB im Jahr 1964, zum Zweiten die Beziehung Eddings zu Hellmut Becker, der das MPIB zwischen 1963 und 1981 leitete. Edding vertrat dort bis 1977 die Abteilung für Bildungsökonomie beziehungsweise diesen Forschungsbereich (zu Edding Behm/Reh 2016: 110ff., Behm 2017: 41ff., Rohstock 2019). Quellenbasis sind Eddings veröffentlichte autobiographische Texte: ${ }^{3}$ Seinen Werdegang schilderte er kurz nach der Emeritierung auf Bitten des Herausgebers der Reihe Pädagogik in Selbstdarstellungen (Edding 1978: 1). Ausführlicher wurde er gut ein Jahrzehnt später in seiner Autobiographie (Edding 1989/überarb. 2000). Veranlasst durch Gespräche im MPIB über „Lebensläufe“ und durch seinen 80. Geburtstag (Edding 2000: 13), verfasste er diese mit Akzent auf dem politischen Aspekt seines Lebens.

\subsection{Von der HIPF an das MPIB - Organisationswechsel als Zeichen von Illoyalität?}

Seit Ende 1959 war Edding Inhaber der ersten westdeutschen Professur für Bildungsökonomie an der Hochschule für Internationale Pädagogische Forschung in Frankfurt am Main. Fast unmittelbar nach Gründung des MPIB 1963 wechselte er jedoch nach Berlin, obwohl

1 Gegründet wurde das MPIB als Institut für Bildungsforschung in der Max-Planck-Gesellschaft (IfB); erst 1971 erhielt es den Status eines Max-Planck-Instituts (vgl. Kant/Renn 2013). Im Folgenden wird aus Gründen der Pragmatik durchgängig vom MPIB gesprochen.

2 Für die Loyalitätsthematik waren für mich die zwischen 2014 und 2017 geführten Gespräche mit ehemaligen Kolleg*innen Eddings am MPIB aufschlussreich. Dr. Cornelia Edding danke ich zudem stellvertretend herzlich für die Gespräche über ihren Vater.

3 Zur methodologischen Problematik von Selbstzeugnissen z.B. Rutz 2002. 
das MPIB zur HIPF in direkter Konkurrenz stand und anfangs die Existenz der Frankfurter Einrichtung bedrohte (dazu Behm 2017: 58ff.). Edding erläuterte:

\begin{abstract}
„Gesicherte Unabhängigkeit, Mittel für größere Projekte und enge interdisziplinäre Zusammenarbeit waren die Wünsche für die Bildungsforschung, die mir nach meinen Kieler Jahren am dringlichsten erschienen. Meine Hoffnung, sie als Professor in Frankfurt erfüllt zu finden, wurde enttäuscht. [...] Ich suchte nach einer Institution, die neuartige, auch ärgerliche Forschung tragen könnte [... und folgte] einem Ruf an das Institut für Bildungsforschung, das Hellmut Becker im Rahmen der Max-Planck-Gesellschaft in Berlin gründete" (Edding 1978: 16ff.). ${ }^{4}$
\end{abstract}

Dieser Darstellung nach orientierte Edding seinen Organisationswechsel ausschließlich an dem überformenden Ziel der Verbesserung seiner Forschungsmöglichkeiten, die er in Frankfurt getestet und für ungenügend befunden hätte. Die Praxis von ,Personal in Bewegung ' ist allerdings für die Wissenschaft quer zu disziplinären Feldern charakteristisch und wird kaum als Illoyalität gegenüber dem Arbeitgeber gedeutet. Im Gegenteil werden der Ruf und die Bewegung an andere, zumal an ressourcenstarke Einrichtungen als der Reputation der Wissenschaftler*in förderlich betrachtet. Nimmt man etwa das von Robert Merton, einem der Pioniere der US-amerikanischen Wissenschaftssoziologie in den 1930er und 1940er Jahren typisierte „ethos“ moderner Wissenschaft in demokratischen Gesellschafen als Maß, die ihrem obersten Ziel nach der „extension of certified knowledge“ verpflichtet sei (Merton, 1942/1973: 270), wäre Eddings Wechsel der Organisation im Dienst der Verbesserung seiner Forschungsbedingungen geradezu mustergültig. Loyalität gegenüber der HIPF würde dagegen einer so verstandenen Wissenschaft zuwiderlaufen, da eine solche Treuebezeugung, kontrafaktisch gefasst, im Extrem zum Verbleib Eddings in der Frankfurter Einrichtung geführt hätte, einer gegenüber einem Max-Planck-Institut markant forschungsschwächeren Organisation.

Allerdings zeigt der Blick in weitere Quellen, dass bei dem prestigeträchtigen Wechsel nach Berlin der Faktor der Loyalität dennoch eine wichtige Rolle spielte und zwar im Sinne der Vermeidung des Eindrucks von Illoyalität. Denn was Edding autobiographisch nicht offenlegte, aber Briefe und Planungsunterlagen für das MPIB im Archiv der Max-Planck-Gesellschaft (AMPG) zeigen: Er war nicht nur von der minderen wissenschaftlichen Qualität der HIPF keineswegs überzeugt (vgl. Behm 2017: 59). Darüber hinaus veränderte Edding in seiner Rückschau den Ablauf der Ereignisse und seine eigene Rolle darin. So war er bereits in die frühen Planungen für das MPIB involviert gewesen und hatte erheblich dazu beigetragen, dass die Berliner Einrichtung überhaupt gegründet werden konnte (s. ebd., bes.: 46). Sein dortiger Direktorposten war in diesem Kontext seit Ende der 1950er Jahre vereinbart worden ${ }^{5}$ und die Zeit in Frankfurt trug somit von Anfang an den Charakter einer Zwischenstation. In seinem Rückblick überging Edding jedoch diese Zusammenhänge und bemühte

4 Vor der Frankfurter Zeit hatte Edding seit 1948 am Institut für Weltwirtschaft (IfW) der Universität Kiel gearbeitet. Mit ,ärgerliche[r] Forschung“ meinte er seine am IfW begonnenen statistisch-vergleichenden Studien im Bildungsbereich, die die Kultusministerien zu verhindern gesucht hätten (Edding 1978: 14f.).

5 Vgl. u.a. AMPG, II. Abt., Rep. 43, Nr. 1 (Korrespondenz); zur Besetzung des Direktorats der Abteilung für Bildungsökonomie durch Edding s. etwa AMPG, III. Abt., ZA 80 (Nachlass Becker), Kasten 2 (Institutsplan von 1961, hier: Anhang, S. 3)/der Nachlass befindet sich inzwischen im Geheimen Staatsarchiv Preußischer Kulturbesitz. Auch Eddings Tochter erinnert, dass die Familienplanung bereits vor dem Wechsel nach Frankfurt auf Berlin hin ausgerichtet war (E-Mails von Dr. Cornelia Edding an die Verfasserin vom 14. und 25.02.2019). 
sich stattdessen - unter Nutzung wirkmächtiger Darstellungskonventionen wissenschaftlichen Erfolgs -, nicht einmal den Verdacht von Illoyalität aufkommen zu lassen.

Dass ihn die Sorge um eine entsprechende Zuschreibung damals tatsächlich umtrieb, belegt eine Bemerkung in einem Brief an einen Beamten der niedersächsischen Kultusverwaltung. Diesem gegenüber äußerte Edding 1962 explizit die Befürchtung, an der HIPF für „,untreu“" gehalten zu werden (nach Mast 1989: 69). Auf Seiten der HIPF wiederum kritisierte der Bildungssoziologe Eugen Lemberg noch 1973 mit implizitem Bezug auf Edding und dem mitschwingenden Vorwurf der Illoyalität, dass zur Zeit der Bedrohung durch das MPIB ,die ersten Ratten schon das sinkende Schiff verließen“. ${ }^{6}$ Beide Äußerungen weisen darauf hin, dass die Loyalitätsthematik durchgängig mit mehr oder minder starken, zum Teil sozialpsychologisch erklärbaren Gefühlen verbunden war. So standen komplementär zu Eddings Furcht beziehungsweise seiner Angst vor Ausgrenzung der Ärger oder die Kränkung des ,verlassenen“ (oder, verratenen') Organisationsmitgliedes.

\subsection{Edding und Becker am MPIB - Loyalität gegenüber dem Förderer und Institutsleiter}

Eddings Schilderung seines Wechsels von Frankfurt nach Berlin verweist bereits im Hintergrund auf seine Beziehung zu Hellmut Becker, Gründungsdirektor des MPIB (zu Becker z.B. Singer/Frevert 2014), die auf mehreren Ebenen asymmetrisch war. Becker hatte seit Eddings Arbeitsbeginn in Berlin die Position des leitenden Institutsdirektors inne. ${ }^{7}$ Zudem nahm er Edding gegenüber in gewisser Weise die Rolle eines Förderers ein, da er den Bildungsökonomen in die Planungen für das MPIB einbezogen, ihn an das Institut geholt und ihm damit eine hervorragende Position innerhalb der westdeutschen Forschungslandschaft eröffnet hatte. Für das Ungleichgewicht der Beziehung grundlegend war zudem der große Abstand im sozialen und kulturellen Kapital, das der Ministersohn Becker in hohem Maße besaß, während Edding in einem Kieler Pfarrhaus in vergleichsweise schlichten Verhältnissen aufgewachsen war und diesen Abstand der Erinnerung der Tochter nach auch als solchen empfand. ${ }^{8}$

Welche Rolle spielte für diese Beziehung Loyalität? Auf den ersten Blick geben die autobiographischen Texte Eddings, in denen die loyale Dankesbezeugung gegenüber wissenschaftlichen Förderern, Weggefährten und ,Vorgesetzten“ eigentlich ein erwartbarer Topos wäre, kaum Hinweise. So äußerte Edding zwar seine „Dankbarkeit“ gegenüber den Instituten, an denen er nach 1945 tätig war, pauschal auch gegenüber dem MPIB (Edding 2000: 106). Darüber hinaus aber kommt die Berliner Einrichtung sowohl im Text von 1989 als auch in der überarbeiteten Fassung von 2000 kaum vor. 1978 wiederum hatte Edding die Arbeitsorgani-

6 [Lemberg, Eugen (1973):] Meine Erinnerungen an die Hochschule (später Deutsches Institut) für Internationale Pädagogische Forschung [Anlage zum Brief Lembergs an Erich Hylla vom 21.02.1973]. DIPF/BBF, Institutsarchiv, Best. 300 (Institutsgeschichtliche Sammlung), Nr. 2 [masch./unpag.].

7 Zu dessen Zuständigkeiten und Kompetenzen vgl. hier nur $\S 3$ der ersten Satzung des MPIB, die zum 01.01.1964 in Kraft trat. AMPG, II. Abt., Rep. 66, 581.

8 Edding 1978: 3-5 und Gespräch mit Dr. Cornelia Edding/27.10.2016. 
sation am MPIB etwas eingehender und auch kritisch dargestellt (Edding 1978: 18ff.). Becker selbst jedoch wurde nur an zwei Stellen eher beiläufig und verhalten positiv erwähnt (Edding 1978: 18, 20). In der Autobiographie verzichtete Edding dann auf jegliche Wertung und konstatierte nur noch nüchtern Beckers Funktionen („Initiator dieser Gründung“, Moderator im Ettlinger Kreis, vgl. Edding 2000: 92, 104). Die Beziehung zu Becker bleibt in den Rückschauen daher merkwürdig absent. Erneut eröffnet erst die Sichtung weiteren historischen Materials unter dem Aspekt der Loyalität eine Deutung dieses Umstands. Vorweggenommen ist dabei zu vermuten, dass Edding seine Loyalität Becker gegenüber gerade in Form dieser De-Thematisierung demonstrierte. Darauf weist jedenfalls ein kursorischer Blick in die Geschichte des MPIB hin.

Am MPIB ging es in der Zeit unter Leitung Beckers besonders um die Aufgabe, eine ,innovative“ Bildungsforschung zu etablieren, die in der interdisziplinären Arbeit ihr Zentrum haben sollte (vgl. zur Gründung und Organisation des MPIB Behm 2017, zur Geschichte z.B. Wiarda 2016). Ein vertrauliches Memo Eddings für Becker vom Mai 1969, in dem er vom „Flickwerk der etablierten Projekte“ sprach (nach Wiarda 2016: 184), zeigt jedoch, dass aus der damaligen Sicht Eddings gerade dieses Ziel verfehlt zu werden drohte. Auch Martin Wiarda rekonstruiert in seiner auf Zeitzeugeninterviews aufbauenden Geschichte des MPIB erhebliche Missstände in Konzeption und Organisation der Forschung, die sich über den Zeitraum von Beckers Führung hinweg allmählich kumuliert und letztlich zu der bislang gravierendsten Zäsur in der Institutsgeschichte, der Berufung von Paul B. Baltes zum Institutsleiter 1980, geführt hätten (Wiarda 2016). ${ }^{9}$ Gleichzeitig arbeitet er eine erhebliche Dominanz Beckers und seines Führungsstils für die Institutsentwicklung und den Arbeitsalltag heraus und weist diesem damit für die damaligen ,Erfolge' und ,Misserfolge' des MPIB eine zentrale Verantwortung zu. Hält man sich die eingangs zitierten Erwartungen Eddings an die Bildungsforschung vor Augen, so musste die Institutsentwicklung bis 1969 diesen Ansprüchen mithin klar zuwiderlaufen und eine kritische Position gegenüber Becker begründen. ${ }^{10}$ Damit stimmt überein, dass Edding Becker in der Erinnerung seiner Tochter skeptisch und sogar ablehnend gegenüberstand, festgemacht an dem vielfach mit nach Hause gebrachten Ärger des Vaters über das Verhalten des Institutsleiters. ${ }^{11}$

Vor allem im Hinblick auf die Institutsentwicklung wäre Kritik an Becker also in Absicht auf dessen Führungsrolle und Verantwortung als Leiter des MPIB sachlich angemessen und begründet gewesen - das umso mehr in einer nachlaufenden Autobiographie, die Edding zudem explizit auf die Revision der Entwicklung der Bildungsforschung und -politik in der Bundesrepublik hin ausgerichtet hatte (Edding 2000: 13). Ärger und Kritik sparte Edding allerdings nicht nur in diesen Rückblicken aus, sondern äußerte sie nach Erinnerung von ehemaligen Kollegen auch institutsöffentlich so gut wie nie. Vielmehr wird sein Verhalten übereinstimmend als zurückhaltend, wenn auch nicht ohne punktuelle, überraschende Widerstände, beschrieben. Obwohl selbst MPIB-Direktor und auf nationaler wie internationaler Ebene

9 Baltes wurde als Nachfolger Beckers berufen. Mit seiner Leitung waren einschneidende Veränderung in Forschungsausrichtung, -organisation und Führung verbunden (s. Wiarda 2016: bes. Kap. 4.5, 4.6 und 5.4.1). Der Umstand, dass der wissenschaftlich kaum profilierte Becker das Institut fast 20 Jahre leiten, in der Max-Planck-Gesellschaft (MPG) sozialwissenschaftlich Neuland erschließen und dem MPIB bis Anfang der 1970er Jahre einen hohen bildungspolitischen Einfluss sichern konnte, steht zur Forschung noch aus.

10 Angemerkt sei, dass die 1969 erfolgte Aufhebung der Abteilungsstruktur des MPIB innerhalb der MPG durchaus als revolutionär gelten konnte. ,Erfolg ${ }^{6}$ oder ,Misserfolg' des MPIB sind im vorliegenden Text aber nicht das Thema.

11 Gespräch mit Dr. Cornelia Edding/02.11.2016. 
als Experte gefragt, habe Edding Becker aber zumeist die Bühne überlassen. ${ }^{12}$ Das galt vom Duktus her offenbar auch für sein Verhalten in der Leitungskonferenz des MPIB, die regelmäßig unter Ausschluss der Institutsöffentlichkeit stattfand, und die vom organisationalen Zweck her den Raum für Diskussionen und auch für scharfe Kritik hätte bieten können. ${ }^{13}$ In Kenntnis dieser gesammelten Hinweise lassen sich die autobiographischen Texte Eddings, wie eingangs angedeutet, daher gerade aufgrund ihrer Leerstellen in Bezug auf Becker als Ausweise der Loyalität gegenüber dem ehemaligen Institutsdirektor und Vorgesetzten interpretieren, der auf diesem Wege öffentlich von jeglicher Kritik ausgenommen wurde.

\subsection{Zur Funktionalität von Loyalität in wissenschaftlichen Feldern - eine erste Zwischenbilanz}

Allgemeiner auf die Funktionalität von Loyalität in Wissenschaftsfeldern gewendet, richtete sich die Regulierungskraft von Loyalität nicht nur - wie am Beispiel von Eddings Organisationswechsel kontrafaktisch durchgespielt - auf die Fixierung von Bewegung im physischen Sinn. Wie an Eddings loyaler Haltung gegenüber Becker am MPIB und in seinen autobiographischen Texten gezeigt, bedingte sie zudem im immateriellen Sinn die Bindung von Denkbewegungen. Loyalität wirkte demnach latent darauf, ein vorgeblich umfassend geltendes Grundprinzip von Wissenschaft zu unterwandern. Denn die Zurückhaltung von Kritik richtet sich letztlich gegen eine der zentralen (Selbst-)Zuschreibungen moderner Wissenschaft: die methodisch abgesicherte, an keiner Grenze Halt machende freie Denkbewegung, die an der freien Äußerung von Kritik ihren Probierstein findet. Daran ließe sich weiterführend zum einen die Frage anschließen, inwieweit die skizzierte auffällige sozial-kulturelle Asymmetrie der Beziehung zwischen Edding und Becker im Hinblick auf Loyalitätserwartungen und -bezeugungen ihren Anteil hatte. Zum anderen wäre die Bedeutung organisationaler Faktoren in den Blick zu nehmen. So ist es mehr als naheliegend, dass die organisationale Kultur der Max-Planck-Gesellschaft (MPG) als Verstärker auf die genannten Asymmetrien wirkte. $\mathrm{Zu}$ denken ist besonders an das sogenannte Harnack-Prinzip, das seit Gründung der Vorläuferorganisation der MPG, der Kaiser-Wilhelm-Gesellschaft (KWG), wirksame organisationale Leitbild der MPG (s. z.B. Laitko 1996). Zumindest steigerte die damit programmatisch wie habituell verbundene Überhöhung der hervorragenden, damals primär männlich gedachten Forscherpersönlichkeit, der weitgehend Carte Blanche gegeben werden sollte, Status und gegebenenfalls Unangreifbarkeit der Institutsleiter noch zusätzlich.

Methodisch kommt die Historiker*in bei diesen Überlegungen allerdings in ein schwieriges Fahrwasser. So sind die Deutungen zum Ersten nicht unproblematisch, da Edding im letztgenannten Fall sein Verhalten nicht explizit als loyal (oder treu) bezeichnete oder andere dies taten und die Forscher*in also ihr Verständnis von Loyalität und ihre eigene Felderfah-

12 Z.B. Gespräche mit Prof. Dr. Klaus Hüfner/30.03.2016, Dr. Michael Jenne, zeitweise Assistent der Institutsleitung/06.08.2015 oder Prof. Dr. Ingo Richter/16.09.2016.

13 Gespräch mit Dr. Michael Jenne/06.08.2015. Als ,heiß` ausgetragener Konflikt wurde in den Zeitzeugengesprächen v.a. der vorzeitige Austritt Eddings aus der Bildungskommission des Bildungsrats 1972 erinnert (z.B. Gespräch mit Prof. Dr. Klaus Hüfner/30.03.2016; s. ebf. Edding 1978: 25, dort ohne Erwähnung Beckers). 
rung in das Quellenmaterial hineinträgt. Zum Zweiten hat sie, wenn Loyalität unterhalb formalisierter Regeln thematisch wird, generell ein Quellenproblem. Denn benötigt würde (bislang kaum gesuchtes) Material, das - wie der Brief Lembergs oder die Erinnerung der Tochter Eddings - auf Verhaltenserwartungen und Gefühle von Wissenschaftler*innen schließen ließe. Damit erst könnten die offenbar primär verdeckt ablaufenden Praktiken und informellen Regeln sowie die Kommunikation über Regelverstöße im Hinblick auf Loyalität weiter zugänglich gemacht werden. Hiermit verbunden ist zum Dritten die Frage, inwieweit sich die Forscher*in bei ihrer Suche nach solchem offenbar ,ehrenrührigen' Material selbst sanktionsfrei bewegen könnte, denn sie unterläge ebenfalls den Regulierungsmechanismen von Loyalität in den zumeist miteinander kommunizierenden disziplinären Feldern. Zum Vierten ist solch ein emotional geladenes, subtiles und gegebenenfalls chiffriertes Material schwer interpretierbar. Wenn die Schlüsse also eine größere Tragfähigkeit beanspruchen wollten, wären sie mit theoretischen (Hilfs-)Modellierungen aus den Sozialwissenschaften zu verbinden, besonders in Bezug auf die Regulierung wissenschaftlicher Felder und auf die, in der Einleitung angerissene, Konzeption von Loyalität.

\section{$3 \quad$ Hellmut Becker - Loyalität gegenüber Elitezirkeln und Intellektuellen des NS-Regimes}

Der bekannte Publizist und Jurist Alexander Kluge hat Hellmut Becker in seinem Werk Chronik der Gefühle ein kurzes Porträt gewidmet. Darin versetzt Kluge seinen Förderer der 1950er Jahre zurück in den Zweiten Weltkrieg, in den Russlandfeldzug und in die 1. GebirgsDivision, als deren Mitglied Becker im Herbst 1941 schwer verwundet wird. Kluge beschreibt wie Becker - durch die Bestimmtheit des auf russischem Boden liegenden, hilflosen, verwundeten Körpers gewissermaßen , in die Welt gesetzt ${ }^{\star}$ - zwar die „,hochräumigen Unbestimmtheiten" abstreift, die ihn als Sohn des ehemaligen Kultusministers Carl Heinrich Becker kennzeichnen und offenbar peinigen. Kluge seziert aber auch messerscharf, dass der Realismus, der Becker nach Krieg und Verwundung zugeschrieben wird, ${ }^{14}$ nicht ungebrochen, nicht frei von Hypostasierungen ist, die ihm sein „gesellschaftlicher Rang“ auferlegt. Kluge schreibt hierzu: „So wie dieser Vater kann der Sohn nicht werden. Es ist aber auch ausgeschlossen, dass er sich einen geringeren Rang zuschreibt“. Das „elitäre Bewusstsein“, das Becker auf diese Weise den Weg zur Wirklichkeit versperrt, versteckt er zunächst unbemerkt ,,in der Gangart eines gewöhnlichen Gebirgsjägers“. Aber die Ahnung, „etwas ganz Besonderes“ zu sein scheint auch später, als Becker als körperlich Versehrter und realistisch „UMGEWERTETER“ in sein Leben zurückmarschiert, immer wieder aufgewallt zu sein (alle Zitate nach Kluge 2000: 373f.). ${ }^{15}$

Die Ironie dieser ebenso klug gesponnenen wie gut erzählten Geschichte besteht nun allerdings darin, dass die 1. Gebirgs-Division mitnichten die „gewöhnliche“ Wehrmachtseinheit war, als die Alexander Kluge sie ausflaggt. Der Truppe, in der Becker sein qua Herkunft vorhandenes Elitebewusstsein vor den scheinbar einfachen „Kameraden“ verstecken konnte, haftete selbst der Nimbus einer Eliteeinheit an: Adolf Hitler persönlich bezeichnete den Wehrmachtsverband als ,seine Garde-Division“ (Meyer 2008: 267). Womöglich liegt hier, in dieser gleichsam doppelten Zugehörigkeit Beckers zu Eliten - der bildungsbürgerlichen,

14 Kluges Becker-Porträt trägt den bezeichnenden Titel: „Er gilt als Realist“ (Kluge 2000).

15 Herv. i. Orig. 
intellektuellen des Elternhauses und der militärischen der Wehrmacht - der Schlüssel zum Verständnis von Beckers langjähriger Loyalität gegenüber Eliten- und Intellektuellenzirkeln des NS-Regimes - eine Loyalität, die ihn, wie zu zeigen sein wird, in der Nachkriegszeit nicht nur Kriegsverbrecher verteidigen, sondern auch zu einem der einflussreichsten Gatekeeper des wissenschaftlichen und wissenschaftspolitischen Feldes der frühen Bundesrepublik aufsteigen ließ (Frei 1996: 178).

\subsection{Loyalität vor dem Hintergrund eigener NS-Belastung}

Ähnlich wie Friedrich Edding, für den Loyalität ein Schlüsselthema war, hat auch Hellmut Becker Loyalität in veröffentlichten wie unveröffentlichten Schriften immer wieder - wenn auch oftmals lediglich mittelbar - thematisiert. Loyalität forderte er von anderen ebenso ein, wie er sie selbst aufführte und praktizierte. ${ }^{16}$ Sie war ihm wichtig, eine der „Grundtugenden“, die die Schule zu vermitteln habe (Becker/Liegle 1980: 14). Ähnlich wie die Journalistin Margret Boveri, zu der Becker eine langjährige Freundschaft pflegte, interessierten ihn Menschen, die sich in den Zwiespalt einer „Doppelloyalität“ manövriert und damit die dunkle Kehrseite der Loyalität, den „Verrat“, sei es am eigenen Gewissen oder einem Regime, kennengelernt hatten (Becker 1980: 51ff.). Gut möglich, dass Ernst von Weizsäcker, der als Staatssekretär im Auswärtigen Amt für die Deportation der europäischen Juden in die nationalsozialistischen Vernichtungslager mit verantwortlich zeichnete und den Hellmut Becker als junger Jurist im sog. Wilhelmstraßen-Prozess verteidigte, für Becker einer jener doppelt Loyalen war (ebd.). Die „,beträchtliche Empathie“, mit der sich Becker als Verteidiger in die „Viten von Intellektuellen“ vertiefte, die „zu Massenmördern geworden waren“, erklärt Ulrich Raulff mit dem Möglichkeitshorizont, unter dem sich Beckers eigene Biografie aufspannte: „Mag sein“, so schreibt Raulff,

„dass er in den Wahlen, die sie [die Kriegsverbrecher, Anm. AR] getroffen hatten, Abzweigungen erkannte, die auch an seinem Weg möglich gewesen wären. Ein gnädiges Geschick, eine familiäre Bindung, eine psychische Disposition hatten ihn davor bewahrt, die falsche Richtung einzuschlagen“ (Raulff 2008: 406).

Gut möglich ist aber auch, dass sich Becker durch seine eigene Vita Kriegsverbrechern wie Ernst von Weizsäcker, Otto Ohlendorf oder Martin Sandberger tatsächlich näher als bislang angenommen fühlte und es eben jene Nähe und jenes Zugehörigkeitsgefühl zu einer spezifischen NS-Elite war, die ihn loyal handeln ließen. Wie lässt sich diese Lesart, die die Interpretation Raulffs variiert, plausibilisieren? Von der Erziehungswissenschaft wie von der Geschichtswissenschaft ist bislang kaum beachtet worden ${ }^{17}$, dass Hellmut Becker seit 1937 nicht nur Mitglied der NSDAP (Raulff 2009), sondern von 1935 bis 1937 auch Mitglied des Nationalsozialistischen Kraftfahrkorps (NSKK), von 1936 bis 1937 der Nationalsozialistischen Volkswohlfahrt (NSV), von 1933 bis 1935 der Deutschen Studentenschaft und von

16 Schreiben Hellmut Beckers an Ernst Rudolf Huber vom 23.05.1953, in: Bundesarchiv Koblenz (BArch Koblenz), NL Ernst Rudolf Huber, N1505-246.

17 Einzige erwähnenswerte Ausnahme: Seliger (2016). 
1936 bis 1939 der Reichsdozentenschaft war. ${ }^{18}$ Zwar sagt die bloße Zugehörigkeit zu diesen Organisationen des NS-Regimes allein noch nichts aus über Denken und Handeln eines Mannes in der nationalsozialistischen Diktatur und über seine Loyalität zu NS-Eliten. Gleichwohl dürfen diese Mitgliedschaften auch nicht - wie das bislang der Fall war - gänzlich ausgespart werden, zumal sie in ihrer Dichte das Bild verschieben, das wir von Becker bislang hatten. Er selbst hielt sie nach Kriegsende für immerhin so wichtig, dass er sowohl den Fragebogen für seine Zulassung zur Verteidigung von Ernst von Weizsäcker (Seliger 2016: 536, Trials of War Criminals 1949: 311) als auch den Fragebogen im Rahmen seines Entnazifizierungsverfahrens fälschte. ${ }^{19}$ Nur mit erheblicher Mühe war es Becker überhaupt gelungen, das politische ,Säuberungsverfahren' nach 1945 in die französische Zone zu verlegen. Und auch dort, wo man ihm dank seiner Freundschaft zu Carlo Schmid wohlgesonnen war, wurde er nicht als ,entlastet“", sondern als „Mitläufer“ des NS-Regimes eingestuft. ${ }^{20}$

Darüber hinaus trat Becker bereits 1935/36 eben jener oben erwähnten Elite-Einheit Hitlers bei ${ }^{21}$, die nur wenig später selbst in schreckliche Verbrechen involviert war: Unter ihrem General Hubert Lanz war die 1. Gebirgs-Division im Juni und Juli 1941 mit verantwortlich für Massenexekutionen an Zivilistinnen und Zivilisten in Lemberg/Lwow. Mehrere tausend Menschen, darunter viele Jüdinnen und Juden, fanden so den Tod (Meyer 2008, Schenk 2007, Rossoliński-Liebe 2013). Mindestens sah die 1. Gebirgs-Division dem Morden der später eintreffenden ,gefürchteten Einsatzkommandos 5 und 6 der Einsatzgruppe $C^{\text {“" tatenlos zu }}$ (Meyer 2008: 64). Glaubt man aber Augenzeugenberichten, dann sind auch Wehrmachtsangehörige zu den Mordstätten ,gepilgert‘ und haben diese zu Propagandazwecken fotografiert. Zudem geben Beobachterinnen und Beobachter des Massakers an, dass Soldaten die Pogromstimmung aufgeheizt und sogar selbst gemordet hätten (Aly/Heim 1990).

Zwar ist letztlich nicht zu klären, ob Hellmut Becker in Lemberg war. Allerdings legen jüngst veröffentlichte Dokumente nahe, dass Becker in der Zeit des Lemberger Pogroms im Sommer 1941 in der 1. Gebirgs-Division unter Hubert Lanz gedient hat und er sich dem ehemaligen Jura-Studenten aus Entringen offenbar verbunden fühlte: 1947 sagte Becker für ,seinen' Kommandanten Hubert Lanz aus und bescheinigte ihm unter Eid ehrenhaftes Verhalten gegenüber russischen Kriegsgefangenen. ${ }^{22}$ Hubert Lanz wurde trotz Beckers Eintreten

18 Fragebogen des Staatskommissariats für Politische Säuberungen vom 05.12.1945, in: Staatsarchiv Sigmaringen, Wue 13 T 2_2016-007.

19 ebd. Im ,Entnazifizierungsbogen' taucht die NSDAP-Mitgliedschaft nicht auf; in dem Fragebogen zur Zulassung zur Verteidigung Ernst v. Weizsäckers werden sowohl NSDAP-Mitgliedschaft als auch die Mitgliedschaften in den anderen NS-Organisationen nicht erwähnt. Das war ein klarer Verstoß gegen die Direktiven der Militärregierung und hätte geahndet werden müssen. Becker konnte aber offenbar beschwichtigen. So heißt es: „Dr. Becker has suggested that the Tribunal grant this application with the provision that any complications or punishment which may result from this falsification be postponed until the case is over with"(Trials of War Criminals 1949: $311)$.

20 ebd.

21 Schreiben der Deutschen Dienststelle an die Autorin vom 18.08.2016; Anschriftenverzeichnis der ehemaligen deutschen Gebirgstruppe (13. Geb. Jäg. Reg. 99), in: Geheimes Staatsarchiv Preußischer Kulturbesitz (GStA PK), VI HA, NL Hellmut Becker, 1622. Das „Schießbuch“ von Hellmut Becker für „Pistole“ gibt an, dass Becker 1935/36 dem Gebirgsjägerregiment 99, 12. M.G. Kompanie, angehörte: GStA PK, VI. HA, NL Becker, C. H., 6292.

22 Die Angaben stammen aus Dokumenten des Nuremberg Trials Project der Haravard University: Affidavit concerning General Lanz's concern for the medical care of Russian POWs in 1941 
nach 1945 zu 12 Jahren Haft wegen der Teilnahme an Kriegsverbrechen dramatischen Ausmaßes in Jugoslawien, Albanien und Griechenland verurteilt. ${ }^{23}$

Bei aller Verschiedenheit, die es allein aufgrund von Stellung und Rang zwischen den beiden Männern gegeben haben muss - Lanz war General, Becker einfacher Soldat: Ihre Wege haben sich im Krieg in einer Eliteeinheit der Wehrmacht gekreuzt. Gut möglich ist also, dass Becker Lanz gar nicht als den fundamental Anderen, der zu einem bestimmten Zeitpunkt einen anderen Weg gewählt hatte und ,falsch abgebogen' war, sondern vielmehr als elementaren Teil der eigenen Geschichte betrachtete. Beckers Loyalität Lanz gegenüber könnte so nicht nur als der Versuch gelesen werden, Erwartungen an soldatische Treue zu entsprechen, der sich die Mitglieder der Gebirgsjäger zweifelsohne auf besondere Art und Weise verpflichtet fühlten (Klein 2007, Klein/Mentner/Stracke 2004). Möglicherweise ging es bei Beckers Eintreten für Lanz auch darum, sich der Integrität seines eigenen Lebenswegs zu vergewissern, der mit den Mitgliedschaften in zahlreichen NS-Organisationen und dem frühen und freiwilligen Beitritt zu den renommierten Gebirgsjägern, die später selbst an Kriegsverbrechen teilnahmen, alles andere als unbelastet war.

Im Fall von Martin Sandberger und Otto Ohlendorf liegen die Dinge anders. Beide waren zwar ebenfalls hochrangige Kriegsverbrecher. Martin Sandberger - wie Hellmut Becker Jurist und früherer Assistent des engen Becker-Vertrauten Carlo Schmid in Tübingen - befehligte das Einsatzkommando 1a und gilt als Hauptverantwortlicher für die ,Entjudung' des Baltikums (Frei 1996: insbes.: 297ff.). Ohlendorf, gleichfalls Jurist, war als Befehlshaber der Einsatzgruppe D für den Tod von rund 90.000 Juden verantwortlich und zeichnete sich in den Nürnberger Prozessen durch die schonungslose und detailreiche Beschreibung der Massentötungen aus (Angrick 2003, Richter 2011). Allerdings sind sich nach bisherigem Kenntnisstand Becker, Ohlendorf und Sandberger weder im Krieg noch zuvor begegnet (Raulff 2009, Seliger 2016), ihre Geschichten also nicht auf ähnliche Weise verflochten wie die Beckers und Lanz“. Beckers juristisches Eintreten für beide „Einsatzgruppen-Mörder“ (Rath 2017) erklärt sich vermutlich eher aus einer Gemengelage von Eliteempfinden, Zugehörigkeitsgefühl zu Intellektuellenzirkeln und Loyalität gegenüber Freunden und Förderern wie Carlo Schmid und Richard von Weizsäcker, auf deren Betreiben hin Becker etwa die Verteidigung von Sandberger übernommen hatte (Seliger 2016: 303, Raulff 2009: 404). Insbesondere im Falle Ohlendorfs wird deutlich, dass Becker in ihm zwar zweifelsohne einen „Massenmörder" sah, dem der Tod angesichts der Schwere seiner Taten kaum erspart bleiben konnte. Aber er galt ihm eben auch ,als echter Intellektueller“, dem er sich qua Herkunft und eigenem Identitätsentwurf verbunden fühlte (Raulff 2009: 405).

(14.08.1947) und Transcript for NMT 7: Hostage Case (01.12.1947) und sind abzurufen unter: http://nuremberg.law.harvard.edu/search/?q=Hellmut+Becker\&m=documents\&m=transcripts\&m=photographs (letzter Zugriff 07.03.2019). Darin heißt es: „The following document will be offered as Lanz Exhibit No. 156. and it is Document No. 13, page 38 of Lanz Document Book I. This affidavit also is executed by a common soldier--not by an officer. He was at the time, Corporal, and his name is Hellmut Becker. "

23 Hubert Lanz und seine Elite-Division verübten im September 1943 u.a. das als „Massaker von Kefalonia“ bekannte Kriegsverbrechen der deutschen Wehrmacht an über 5000 italienischen Soldaten, die sich den deutschen Gebirgsjägern bereits ergeben hatten (Meyer 2008). Hieran war Becker zweifelsohne unbeteiligt: er wurde im Herbst 1941 bei Polugorod in Russland durch einen „Oberschenkeldurchschuss und Fußrücken links“ verwundet wurde und im Dezember 1941 ,in die Heimat" transportiert. Schreiben der deutschen Dienststelle an die Autorin vom 18.08.2016. 


\subsection{Die „Nazis", das waren „die anderen“ - Beckers Beeinflussung des wissenschaftlichen Feldes und der westdeutschen Nachkriegsordnung}

Dabei fällt auf: Beckers Loyalität gegenüber NS-Eliten und Kriegsverbrechern zeichnete sich durch seinen Glauben daran aus, dass er keine „Nazis“ verteidigte. Die Mehrheit derer, für die er eintrat, wiesen wichtige biografische Schnittmengen mit Beckers eigener Vita auf - sie waren Juristen und/oder Vorgesetzte, galten als Intellektuelle, gehörten dem Bildungsbürgertum an, waren der Familie oder wichtigen Freunden Beckers im Umfeld des Stefan GeorgeKreises verbunden. Becker sah sich in ihnen vermutlich wie in einem Spiegel - „die Nazis“, das waren „die anderen“. Hellmut Becker gehörte nicht zum engeren Kreis der Georgianer, jenem eingeschworenen Zirkel bürgerlicher Intellektueller, die das geistige Erbe des „Dichterfürsten" Stefan George auch nach seinem Tod weitertrugen (Groppe 2001, Raulff 2009). Aber er war, wie es Eingeweihte ausdrückten, ,,angekreist“, d.h. über Familie und Freunde mit dem engsten Zirkel verbunden und durch seine Ideen beeinflusst (Beßlich 2016: 1069). Mit den Mitgliedern des George-Kreises teilte er das Empfinden, etwas Besonderes, gar auserwählt zu sein. Der Journalist Erich Kuby, der kurz vor Kriegsende Straßburg besuchte, wo Becker als Assistent Ernst Rudolf Hubers wirkte, hat dieses Elitebewusstsein in einer Beobachtung eingefangen: „Diese Kultur- und Wissenschaftsplutokraten tragen ein unsichtbares Schild um den Hals: Wir sind die anderen Deutschen“" (Kuby zitiert nach Raulff 2009: 476).

Dass es „die anderen Deutschen“ ausgerechnet an die Reichsuniversität Straßburg als wissenschaftlichen Außenposten des NS-Regimes verschlagen hatte, dass Becker ausgerechnet bei Ernst Rudolf Huber, dem als „Kronjuristen“ des Dritten Reichs bekannten Staatsrechtler (Rüthers 1988), assistierte, dass Becker nach oder während seiner Straßburger Zeit als juristischer Mitarbeiter in Unternehmen tätig war, die Rüstungsgüter herstellten und Zwangsarbeiterinnen und Zwangsarbeiter rekrutierten - all das tat seinem Empfinden, auf „der anderen“ Seite zu stehen, offenbar keinen Abbruch. ${ }^{24}$ Und in diesem Sinne baute er auch seine Verteidigung auf: In einem Schreiben an die Journalistin Margret Boveri forderte er die Freundin 1948 auf, im Falle Ernst von Weizsäckers noch einmal schärfer die ,prinzipielle Frage des Verhältnisses der Anklage zu den wirklichen Nationalsozialisten“" zu stellen. Und weiter:

„Es müsste einmal ganz offen und direkt die Frage erörtert werden: wer hat das Umbringen der Juden veranlasst und wer hat es ausgeführt, und wer ist für die ganze Sache wirklich verantwort-

24 Schreiben Hellmut Beckers an Ernst Rudolf Huber vom 23. Juli 1945, in: BArch Koblenz, NL Huber, N1505-246. Wenn Becker in oder kurz nach dem Krieg bei Maybach und/oder der Zahnradfabrik Friedrichshafen beschäftigt war (dies legt im Übrigen auch sein Entnazifizierungsbogen nahe), ist er dort mit Zwangsarbeit konfrontiert gewesen. Vgl. Tholander 2001, vgl. auch: https://www.dsk-nsdoku-oberschwaben.de/de/erinnerungswege/bodenseekreis-und-sigmaringen/friedrichshafen-massenlager-fuer-zwangsarbeiter-hochstrasse-heinrich-heine-strasse.html (letzter Zugriff 06.03.2019). Anfragen an und Antworten der Firmen befinden sich im Besitz der Autorin. 
lich. Durch eine solche Betrachtungsweise müsste einmal dem ewigen Heranziehen neuer Gruppen, die angeblich auch daran schuld seien, ein Ende gemacht werden und der Unsinn der hiesigen Anklage auch in diesem Zusammenhang herausgestellt werden." 25

Auch nutzte Becker seine politischen Verbindungen aus, um eine „großzügige Amnestie“ gegenüber den für Kriegsverbrechen Angeklagten zu erwirken. ${ }^{26}$ Hier ging es ihm explizit darum, über die deutsche Politik ,die Alliierten und zwar möglichst hochstehende für den Gedanken einer Amnestie zu gewinnen“ und so eine alliierte „Aktion“ zu initiieren, „die die Menschen aus dem Gefängnis herausbringt, die ihrem Wesen nach nicht hereingehören““. 27 Die Konsequenzen dieses Ansinnens gingen weit über den persönlichen Bereich hinaus: In einer mit ungeheurem Aufwand betriebenen Medienkampagne, für die Becker insbesondere Margaret Boveri, die Wochenzeitung Die Zeit und seine Freundin Marion Gräfin Dönhoff einspannte, versuchte Becker die öffentliche Meinung darüber zu beeinflussen, wer in der bundesrepublikanischen Nachkriegsordnung ,eigentlich' als Kriegsverbrecher und Nationalsozialist zu gelten hatte. Unterstützt wurde er dabei von einer Vielzahl anderer Anwälte, die eine Gruppe von Historikerinnen und Historikern aus Jena vor kurzem treffend als „hochprofessionelle[n] Kriegsverbrecher-Lobby“ bezeichnet hat (Frei u.a. 2019: o. S.). Dieser Lobby ging es darum, Grenzen zu etablieren, anhand derer erst bestimmt werden konnte, wer als Täter und was als Verbrechen beurteilt werden sollte. ${ }^{28}$ Der Versuch Beckers und anderer Juristen, in ihrem Sinne „die verschiedensten kirchlichen und politischen Kräfte zu mobilisieren“, zeigt, wie viel in dieser Frage auf dem Spiel stand: Letztlich wurde hier verhandelt, was den Kern des Nationalsozialismus ausmachte. ${ }^{29}$ Beckers Loyalität gegenüber belasteten NS-Eliten könnte demnach als der Versuch gelesen werden, sich über die rechtliche Beurteilung von Schuld und Unschuld selbst auf Distanz zum Nationalsozialismus zu bringen.

Auch im wissenschaftlichen Feld kam diese Loyalität zum Tragen. So setzte sich Becker für seinen akademischen Lehrer und Vertrauten aus Straßburger Tagen Ernst Rudolf Huber ein, zunächst im Entnazifizierungsverfahren ${ }^{30}$, später auch im Rahmen der Vergabe von

25 Schreiben Beckers an Boveri vom 11.8.1948, in: PA AA, NL 33, Weizsäcker-Prozess, B-Br, Bd. 3, Teil 1 .

26 Schreiben Beckers an Theo Kordt vom 26.3.1949, in: PAAA, NL 33, Weizsäcker-Prozess, Ko-Ku, Bd. 7.

27 ebd.

28 Patrick Bernhard hat in einem anderen, jedoch ähnlich gelagerten Kontext das Verhalten der Staatsanwaltschaften gegenüber den Verbrechen an Tuberkulosekranken ,,als den letztlich erfolgreichen Versuch“ bezeichnet, ,den Nationalsozialismus und die in seinem Namen begangenen Verbrechen einzuhegen, sie auf bestimmte Tatkomplexe, Täter und Tatorte zu begrenzen, auf diese Weise eine mehrfache Distanz zu den Geschehnissen herzustellen und somit letztlich eine bestimmte Form von Normalität zu erzeugen“ (Bernhard, in Vorbereitung 2020).

29 Schreiben Beckers an Theo Kordt vom 26.03.1949, in: PAAA, NL 33, Weizsäcker-Prozess, Ko$\mathrm{Ku}, \mathrm{Bd} .7$.

30 Schreiben Beckers an Huber vom 06.08.1948, in: BArch Koblenz, NL E. R. Huber, N1505-246. 
Lehraufträgen ${ }^{31}$, in Berufungsverfahren ${ }^{32}$ und der Besetzung wissenschaftlicher Schaltstel$\operatorname{len}^{33}$. Als Huber wegen seiner Rolle als NS-Staatsrechtler unter politischen Druck geriet und die Rücknahme eines mühsam erwirkten Lehrauftrags im Raum stand, übte Becker persönlich nicht zu unterschätzenden Druck auf das Stuttgarter Kultusministerium unter Gotthilf Schenkel aus. Seine „fast 4-stündige Unterhaltung mit Min.rat Müller ${ }^{34 ،}$ fasste Becker in einem an Ernst Rudolf Huber adressierten Schreiben für den Freund folgendermaßen zusammen: „Ich glaube aber doch, dass Müller Einiges kapiert hat. Ich habe ihm keinen Hehl daraus gemacht, dass ich jede kränkende Maßnahme Ihnen gegenüber für unverständlich halte und ich habe das Gefühl, dass er bestrebt ist, mir gegenüber nicht illoyal zu sein. “"35

In seiner Zeit als Mitglied des wissenschaftlichen Beirats des Instituts für Zeitgeschichte (IfZ) versuchte Becker darüber hinaus, auch die Grenzen des wissenschaftlich Sagbaren zu konturieren. Als „Deutsches Institut für Geschichte der nationalsozialistischen Zeit“ gegründet (Möller/Wengst 2009), war Becker im Beirat mit Arbeiten konfrontiert, die die NS-Vergangenheit thematisierten. Dass das IfZ in den 1970er Jahren unter der Leitung von Martin Broszat ausgerechnet Becker als Gutachter zu Arbeiten über eben jene Einsatzgruppen bestellte, deren Täter er nach 1945 öffentlichkeitswirksam verteidigt hatte, ist mit Nachlässigkeit nicht zu erklären. ${ }^{36}$ Zwar versuchte Becker entsprechende Arbeiten nicht zu verhindern. Im Gegenteil setzte er sich nach eigenem Bekunden für die Aufarbeitung etwa der „Einsatzgruppenbefehle " ein. ${ }^{37}$ Doch verläuft die Diskriminierung von wissenschaftlichen Positionen ohnehin selten auf plumpe Art und Weise. Becker fuhr vielmehr seine bewährte Strategie: Revisionisten und Apologeten des NS-Regimes kritisierte er scharf. ${ }^{38}$ Mit „(Alt-)Nazis“ wollte er nichts zu tun haben, das waren - einmal mehr - ,die Anderen“. Arbeiten hingegen, die für ihn unbequem gewesen sein dürften, weil sie die mühsam gesetzten Grenzen verschoben und einmal konstruierte Wirklichkeiten in Frage stellten, wusste er mit subtileren Mitteln abzuwehren und abzuwerten: Selbst das IfZ, das der Exklusion von unliebsamen Positionen nicht unbedingt unverdächtig war, zeigte sich „sehr überrascht“ von einem Gutachten Beckers, in dem er die Theorieferne einer Arbeit, die die Taten der Einsatzgruppen aufarbeitete, anprangerte..$^{39}$ Auf diesem angeblichen „Theoriedefizit“ baute er sein gesamtes Gutachten

31 Siehe weiter unten.

32 Schreiben Beckers an Dr. Tula Huber, die Ehefrau Ernst Rudolf Hubers, vom 13.10.1955, in: BArch Koblenz, NL E. R. Huber, N1505-246.

33 Offenbar dachte Becker daran, Huber als Mitglied des Beirats des IfZ vorzuschlagen, weil er ihm „für dieses Institut eigentlich völlig unentbehrlich" schien. Schreiben Beckers an Huber vom 07.05.1952, in: BArch Koblenz, NL E. R. Huber, N1505-246.

34 Gemeint ist der Ministerialrat im Stuttgarter Kultusministerium Gerd Müller, der kurz darauf einen Herzanfall erlitt. Vgl. Schreiben Beckers an Huber vom 10.06.1953, in: BArch Koblenz, NL Ernst Rudolf Huber, N1505-246.

35 Schreiben Beckers an Huber vom 23.05.1953, in: BArch Koblenz, NL Ernst Rudolf Huber, N1505246.

36 Ergebnisprotokoll der Sitzung des Wissenschaftlichen Beirats des Instituts für Zeitgeschichte in München am 07.03.1974, in: GStA PK, VI. HA NL Hellmut Becker, 360.

37 Gutachten Beckers zu Hans-Heinrich Wilhelm, Die Einsatzgruppe A der Sicherheitspolizei und des SD 1941/42, 5.3.1976, in: GStA PK, VI. HA NL Becker, Hellmut, 388.

38 Vgl. etwa Hellmut Becker: Bemerkungen zu der Arbeit von Otto Schweling: „Die deutsche Wehrmachtsjustiz im 3. Reich“, mündlich vorgetragen in der Sitzung des Beiratsausschusses des IfZ am 07.11.1975, aufgeschrieben für die Beiratssitzung am 11.03.1976, in: GStA PK, VI. HA NL Becker, Hellmut, 388.

39 Ergebnis-Protokoll der Sitzung des Wissenschaftlichen Beirats des IfZ in München, 07.03.1975, in: GStA PK, VI. HA NL Hellmut Becker, 388. 
auf, ohne dass deutlich wird, worin der Mehrwert einer solchen theoretischen Fundierung hätte liegen können. Schließlich schickte er sich sogar an, „,vor einer Publikation in der Reihe des Instituts für Zeitgeschichte [zu] warnen“: Die Dissertation kanzelte er als „Erlebnis- und Ereignisbericht“ ab, ,der nicht auf dem Niveau moderner Geschichtsschreibung steht und die Einsatzgruppenberichte mit einer gewissen Distanzlosigkeit verwendet". Die von ihm selbst genannten Stärken der Arbeit, etwa, „dass die Mitbeteiligung der Wehrmacht [an den Massentötungen, Anm. AR] klar herausgearbeitet wird" und die Dissertation auf diese Weise „,nicht zur Verstärkung der Legende von der sauberen Wehrmacht und der schmutzigen SS“ beitrug, konnte das von Becker identifizierte theoretische Versäumnis offenbar nicht aufwiegen. ${ }^{40}$ Die Arbeit, die noch heute als eine der ersten und ,wichtigen“ Studien zu den Einsatzgruppen gilt (Wildt 2002: 32), wurde nach dem Gutachten Beckers erst mit erheblicher Verzögerung publiziert (Krausnick/Wilhelm 1981).

Liest man die Forderung nach „mehr Theorie“ als Forderung nach nüchterner, distanzierter und kühler Wissenschaftlichkeit, bediente Becker damit ein nicht ganz untypisches narratives Muster der Nachkriegszeit. Der Historiker Nicolas Berg hat darauf aufmerksam gemacht, dass in dem „Pathos der Nüchternheit“, das etwa IfZ Direktor Martin Broszat für sich reklamierte (Berg 2002), eine ,unausgesprochene Entlastungssehnsucht“ (Berg 2003: 614) eines in das NS-Regime Verstrickten mitschwang, die sich auch auf Broszats wissenschaftliche Arbeiten auswirkte. Beckers Begründungen für wissenschaftliche Urteile und Entscheidungen im Beirat des IfZ weisen nicht nur eine ganz ähnliche Semantik auf. Naheliegend ist auch, dass für seine Urteile persönliche und biografische Bindungen und Erfahrungen eine Rolle spielten, die Becker als gleichsam unsichtbare Loyalitätsbeziehungen in das wissenschaftliche Feld trug. Seine wiederholten Forderungen nach methodischer Strenge oder Theorieaffinität können jedenfalls nicht darüber hinwegtäuschen, dass für Becker selbst als Gatekeeper offenbar ganz andere Spielregeln galten ${ }^{41}$ : So empfahl er etwa einem Autor ganz unumwunden, in seiner Arbeit stärker deutlich zu machen, ,wie der Wunsch, durch künstlerische und wissenschaftliche Qualität nach Frankreich auszustrahlen, in den letzten beiden Kriegsjahren in Straßburg eine dem Nationalsozialismus ferne geistige Insel entstehen ließ“", deren herausragendes Charakteristikum „Oper, Theater und Konzert“ gewesen sei. ${ }^{42}$ Diese Äußerung Hellmut Beckers ist durch eben jene unkritische Distanzlosigkeit des unmittelbar Involvierten gekennzeichnet, die er in seinen Gutachten wiederholt anprangerte. Sie verweist darauf, dass Becker selbst eine Geschichtsdeutung vorgab, die alles andere als distanziert, nüchtern und rational, sondern von persönlichen Loyalitätsbeziehungen gekennzeichnet war und ganz elementar eine Entlastungsfunktion erfüllte.

40 Gutachten Beckers zu Hans-Heinrich Wilhelm, Die Einsatzgruppe A der Sicherheitspolizei und des SD 1941/42, 05.03.1976, in: GStA PK, VI. HA NL Becker, Hellmut, 388.

41 Bemerkungen zu der Arbeit von Otto Schweling: „Die deutsche Wehrmachtsjustiz im 3. Reich“, 11.03.1976, in: GStA PK, VI. HA NL Becker, Hellmut, 388.

42 Gutachten Hellmut Beckers zu: „Die nationalsozialistische Volkstumspolitik im Elsaß während des Zweiten Weltkriegs von Lothar Kettenacker, 02.09.1969, in: GStA PK, VI. HA NL Becker, Hellmut, 360. 


\title{
3.3 Zur Funktionalität von Loyalität in wissenschaftlichen Feldern - eine zweite Zwischenbilanz
}

Beckers Beispiel zeigt zum einen, dass Loyalität sein politisches und juristisches Denken und Handeln in der Nachkriegszeit entscheidend mitbestimmte. Mit seinem Eintreten für NS-Täter, der geschickten Argumentation für ihre Unschuld und der massiven Beeinflussung des öffentlichen Diskurses sowie der Politik, prägten Becker (und andere) nicht nur maßgeblich das Meinungsklima der Nachkriegszeit (Frei 1996, Frei u.a. 2019, Raulff 2009, Seliger 2016). Becker und die „Kriegsverbrecherlobby“ halfen auch, „,den Deutschen“ eine Erzählung zu liefern, die sich über viele Jahrzehnte bequem glauben ließ: Schuld an den Verbrechen des NS-Regimes waren dieser Narration zufolge Hitler und seine Schergen sowie eine relativ begrenze Zahl von ,wirklichen“ Nationalsozialisten (vgl. dazu auch: Czollek 2018). Nationalsozialismus - und das suggerieren auch die angeführten Zitate - wurde in dieser Erzählung als etwas Wesenhaftes begriffen, das die Mehrheit der Deutschen eben „ihrem Wesen nach“ schlicht nicht teilte.

Zum anderen spricht vieles dafür, dass Beckers Loyalität gegenüber Intellektuellen und Eliten des NS-Regimes auch Einfluss auf sein wissenschaftliches Urteil und die von ihm angestrengte Personalpolitik hatte. In letzter Konsequenz waren es auf diese Weise u.a. auch einflussreiche Wissenschaftler und Wissenschaftspolitiker wie Hellmut Becker bzw. ihre Loyalitätsbeziehungen untereinander, die dafür sorgten, dass die „Täterforschung“, die den Holocaust aus seiner Abstraktheit löste und erstmalig konkrete Täter in den Fokus der historischen Erforschung des Nationalsozialismus rückte, erst in den 1990er Jahren in Gang kam (Bajohr 2013).

\section{$4 \quad$ Loyalität als analytischer Begriff? Ein (kurzes) Fazit}

So unterschiedlich Loyalität im Falle Friedrich Eddings und Hellmut Beckers auch gefasst werden muss: Die beiden Beispiele zeigen, dass Loyalität wissenschaftlichen Feldern nicht äußerlich ist, sondern vielmehr ein wichtiger, jene Felder zentral mitkonstituierender Bestandteil. Die im vorliegenden Text untersuchten Beispiele weisen insofern übergreifend darauf hin, dass Denkbewegungen in wissenschaftlichen Feldern auch im - analytisch weiter zu klärenden - Begriff der Loyalität ertragreich und mit selbst-reflexivem Gewinn verhandelt werden können. Dies setzt im Übrigen auch ein Fragezeichen hinter eine der bislang dominanten Selbst-Beschreibungen wissenschaftlicher Felder: ihre vorgebliche Autonomie.

\section{Archiv- und Literaturverzeichnis}

\author{
Archiv der Deutschen Dienststelle (WASt) \\ Archiv der Max-Planck-Gesellschaft (AMPG) \\ AMPG, II. Abt., Rep. 43, Nr. 1 (Korrespondenz), 1958-1961 \\ AMPG, II. Abt., Rep. 66 (Generalverwaltung: Institutsbetreuung), 581 \\ AMPG, III. Abt. ZA 80 (NL Becker), Kasten 2 (jetzt im GStA PK) \\ Bundesarchiv Koblenz (BArch Koblenz) \\ BArch Koblenz, NL Ernst Rudolf Huber, N1505-246
}


DIPF/BBF, Institutsarchiv

DIPF/BBF, Institutsarchiv, Best. 300 (Institutsgeschichtliche Sammlung), Nr. 2.

Geheimes Staatsarchiv Preußischer Kulturbesitz (GStA PK)

GStA PK, VI HA, NL Hellmut Becker, 1622

GStA PK, VI. HA NL Hellmut Becker, 360

GStA PK, VI. HA NL Hellmut Becker, 388

GStA PK, VI. HA, NL Becker, C. H., 6292

Politisches Archiv des Auswärtigen Amts (PA AA)

PA AA, NL 33, Weizsäcker-Prozess, B-Br, Bd. 3, Teil 1.

PA AA, NL 33, Weizsäcker-Prozess, Ko-Ku, Bd. 7.

Staatsarchiv Sigmaringen (StA Sigmaringen)

StA Sigmaringen, Wue 13 T 2 2016-007

\section{Literatur}

Aly, Götz/Heim, Susanne (1990): Vordenker der Vernichtung. Auschwitz und die deutschen Pläne für eine neue europäische Ordnung. Hamburg: Hoffmann und Campe.

Angrick, Andrej (2003): Besatzungspolitik und Massenmord. Die Einsatzgruppe D in der südlichen Sowjetunion 1941-1943. Hamburg: Hamburger Edition.

Bajohr, Frank (2013): Neuere Täterforschung, Version: 1.0, in: Docupedia-Zeitgeschichte, 18.06.2013 (http://docupedia.de/zg/bajohr_neuere_taeterforschung_v1_de_2013; DOI: http://dx.doi.org/10.1 4765/zzf.dok.2.243.v1 [Zugriff 04.09.2019].

Becker, Hellmut/Liegle, Ludwig (1980): Israel - Erziehung und Gesellschaft. Stuttgart: Klett-Cotta.

Becker, Hellmut (1980): Auf dem Weg zur lernenden Gesellschaft. Personen, Analysen, Vorschläge für die Zukunft. Stuttgart: Klett-Cotta.

Behm, Britta (2017): Zu den Anfängen der Bildungsforschung in Westdeutschland 1946 - 1963. Ein wissensgeschichtlicher Blick auf eine ,vergessene" Geschichte. In: Reh, Sabine/Glaser, Edith/Behm, Britta/Drope, Tilman (Hrsg.): Wissen machen. Beiträge zu einer Geschichte erziehungswissenschaftlichen Wissens in Deutschland zwischen 1945 und 1990. (Zeitschrift für Pädagogik, 63. Beiheft). Weinheim/Basel: Beltz, S. 34-69.

Behm, Britta/Reh, Sabine (2016): (Empirische) Bildungsforschung - notwendig außeruniversitär? Eine Sondierung der Geschichte westdeutscher Bildungsforschung am Beispiel des Deutschen Instituts für Internationale Pädagogische Forschung (DIPF). In: Baumert, Jürgen/K.-J. Tillmann, KlausJürgen (Hrsg.): Empirische Bildungsforschung. Der kritische Blick und die Antwort auf die Kritiker. (Zeitschrift für Erziehungswissenschaft, 19/Suppl. 1). Wiesbaden: Springer VS, S. 107-127.

Berg, Nicolas (2002): Die Lebenslüge vom Pathos der Nüchternheit. Subjektive jüdische Erinnerung und objektive deutsche Zeitgeschichtsforschung? Joseph Wulf, Martin Broszat und das Institut für Zeitgeschichte in den sechziger Jahren. In: Süddeutsche Zeitung, Nr. 163 vom 17.07.2002, o. S.

Berg, Nicolas (2003): Der Holocaust und die westdeutschen Historiker: Erforschung und Erinnerung. Göttingen: Wallstein.

Bernhard, Patrick (in Vorbereitung 2020): Die ausgebliebene Aufarbeitung: Nationalsozialistische Verbrechen an Tuberkulosekranken und ihre weitgehende Nichtahndung nach 1945. In: ZeitRäume: Potsdamer Almanach des Zentrums für Zeithistorische Forschung (unv. Manuskript). 
Beßlich, Barbara (2016): Einführende Bemerkungen, in: Aurnhammer, Achim/ Braungart, Wolfgang/ Breuer, Stefan/ Oelmann, Ute (Hrsg.): Stefan George und sein Kreis: Ein Handbuch, Band 1. Berlin/Boston: de Gruyter, S. 1069-1072.

Connor, James (2007): The Sociology of Loyalty. Boston: Springer VS.

Czollek, Max (2018): Desintegriert Euch! Berlin: Hanser.

Frevert, Ute (2009): Was haben Gefühle in der Geschichte zu suchen? In: Geschichte und Gesellschaft, 35, S. 183-208.

Edding, Friedrich (1978): [Selbstdarstellung]. In: Pongratz, Ludwig J. (Hrsg.): Pädagogik in Selbstdarstellungen. Band. 3. Hamburg: Felix Meiner, S. 1-34.

Edding, Friedrich (1989): Mein Leben mit der Politik. Berlin: Max-Planck-Institut für Bildungsforschung.

Edding, Friedrich (2000): Mein Leben mit der Politik 1914-1999. Teilhabe an der Entwicklung bildungspolitischen Denkens. Überarb. Neuaufl. Berlin: (Max-Planck-Institut für Bildungsforschung).

Frei, Norbert/Maubach, Franka/Morina, Christina/Tändler, Maik (2019): Zur rechten Zeit. Wider die Rückkehr des Nationalsozialismus. Berlin: Ullstein (e-book).

Frei, Norbert (1996): Vergangenheitspolitik. Die Anfänge der Bundesrepublik Deutschland und die NS-Vergangenheit. München: Beck.

Gläser, Jochen (2006): Wissenschaftliche Produktionsgemeinschaften. Die soziale Ordnung der Forschung. Frankfurt/M./New York: Campus.

Groppe, Carola (2001): Die Macht der Bildung. Das deutsche Bürgertum und der George-Kreis 18901933. Köln/Weimar/Wien: Böhlau-Verlag.

Iser, Mattias (2008): Loyalität. In: Gosepath, Stefan/Hinsch, Wilfried/Rössler, Beate (Hrsg.): Handbuch der Politischen Philosophie und Sozialphilosophie. Band. 1. Berlin: de Gruyter, S. 731-733.

Kant, Horst/Renn, Jürgen (2013), Eine utopische Episode - Carl Friedrich von Weizsäcker in den Netzwerken der Max-Planck-Gesellschaft. Preprint 441. Berlin: Max-Planck-Institut für Wissenschaftsgeschichte.

Kersting, Christa (2008): Pädagogik im Nachkriegsdeutschland. Wissenschaftspolitik und Disziplinentwicklung 1945 bis 1955. Bad Heilbrunn: Klinkhardt.

Klein, Ralph (2007): Das Polizei-Gebirgsjäger-Regiment 18. Massaker, Deportation, Traditionspflege. In: Zeitschrift für Geschichtswissenschaft, 1, S. 41-64.

Klein, Ralph/Mentner, Regina/Stracke, Stephan (Hrsg.) (2004): Mörder unterm Edelweiß. Köln: PapyRossa Verlag.

Kluge, Alexander (2000): Chronik der Gefühle. Band I: Basisgeschichten. Frankfurt/M.: Suhrkamp.

Krausnick, Helmut/Wilhelm, Hans-Heinrich (1981): Die Truppen des Weltanschauungskrieges, Stuttgart: Deutsche Verlagsanstalt.

Kunadt, Susann/Lipinsky, Anke/Löther, Andrea/Steinweg, Nina/Vollmer, Lina (2014): Gender in der Hochschulforschung. Status Quo und Perspektiven. In: die hochschule, 1, S. 106-220.

Laitko, Hubert (1996): Persönlichkeitszentrierte Forschungsorganisation als Leitgedanke der KaiserWilhelm-Gesellschaft: Reichweite und Grenzen, Ideal und Wirklichkeit. In: Vom Brocke, Bernhard/Laitko, Hubert (Hrsg.): Die Kaiser-Wilhelm-/Max-Planck-Gesellschaft und ihre Institute. Studien zu ihrer Geschichte: Das Harnack-Prinzip. Berlin: de Gruyter, S. 583-632.

Lemberg, Eugen (1973): Meine Erinnerungen an die Hochschule (später Deutsches Institut) für Internationale Pädagogische Forschung [Anlage zum Brief Lembergs an Erich Hylla vom 21.2.1973]. DIPF/BBF, Institutsarchiv, Best. 300 (Institutsgeschichtliche Sammlung), Nr. 2 [masch./unpag.].

Mast, Peter (1989): Von der Hochschule zum Deutschen Institut für Internationale Pädagogische Forschung in Frankfurt am Main. 1950-1981. Ein Beitrag zur Geschichte der ,pädagogischen Tatsachenforschung“ in Deutschland. [ohne Ort]/unveröff. Ms.: Bibliothek für Bildungsgeschichtliche Forschung (BBF) 
Merton, Robert K. (1942/1973): The Normative Structure of Science. In: Merton, Robert K. (Ed.): The Sociology of Science. Theoretical and Empirical Investigations. Edited and with an Introduction by Norman W. Storer. Chicago/London, pp. 267-278.

Meyer, Hermann Frank (2008): Blutiges Edelweiß: die 1. Gebirgs-Division im Zweiten Weltkrieg. Berlin: C. H. Links.

Möller, Horst/Wengst, Udo (2009): 60 Jahre Institut für Zeitgeschichte München - Berlin. Geschichte - Veröffentlichungen - Personalien. München Oldenbourg.

Osterkamp, Jana/Schulze Wessel, Martin (Hrsg.) (2017a): Exploring Loyalty. Göttingen/Bristol/Connecticut: Vandenhoeck \& Ruprecht.

Osterkamp, Jana/Schulze Wessel, Martin (2017b): Exploring Loyalty. In: Dies. (Eds.): Exploring Loyalty. Göttingen/Bristol/Connecticut: Vandenhoeck \& Ruprecht, pp. 1-16.

Osterkamp, Jana/Schulze Wessel, Martin (2016): Texturen von Loyalität. In: Geschichte und Gesellschaft, 42, S. 553-573.

Rath, Martin (2017): Rechtsgeschichte: Strafverteidigung in Nürnberg. In: Legal Tribune Online, 05.03.2017. https://www.lto.de/persistent/a_id/22272/ [Zugriff: 07.03.2019].

Raulff, Ulrich (2009): Kreis ohne Meister. Stefan Georges Nachleben. München: C.H. Beck.

Richter, Ilka (2011): SS-Elite vor Gericht. Die Todesurteile gegen Oswald Pohl und Otto Ohlendorf. Marburg: Tectum.

Rohstock, Anne (2019): Vom NS-Statistiker zum bundesrepublikanischen Bildungsforscher. Friedrich Edding und seine Verstrickung in den Nationalsozialismus. In: Rieger-Ladich, Markus/Rohstock, Anne/Amos, Karin (Hrsg.): Erinnern, Umschreiben, Vergessen. Die Stiftung des disziplinären Gedächtnisses als soziale Praxis. Weilerswist: Velbrück Wissenschaft, S. 120-157.

Rossoliński-Liebe, Grzegorz (2013): Der Verlauf und die Täter des Lemberger Pogroms vom Sommer 1941. Zum aktuellen Stand der Forschung. In: Jahrbuch für Antisemitismusforschung, 22, S. 207243.

Rüthers, Bernd (1988): Entartetes Recht: Rechtslehren und Kronjuristen im Dritten Reich. München: C. H. Beck.

Rutz, Andreas (2002): Ego-Dokumente oder Ich-Konstruktion? Selbstzeugnisse als Quellen zur Erforschung des frühneuzeitlichen Menschen. In: zeitblicke 1/2, URL: http://www.zeitenblicke.histori cum.net/2002/02/rutz/index.html [Zugriff: 06.02.2019].

Schenk, Dieter (2007): Der Lemberger Professorenmord und der Holocaust in Ostgalizien. Bonn: Verlag J. H. W. Dietz.

Seliger, Hubert (2016): Politische Anwälte? Die Verteidiger der Nürnberger Prozesse. Baden-Baden: Nomos.

Singer, Kerstin/Frevert, Ute (2014): „100 Jahre Hellmut Becker (1913-2013) - Dokumentation der Ausstellung zu Leben und Werk im Max-Planck-Institut für Bildungsforschung“, Online-Publikation, Oktober 2014. [DOI: 10.14280/08241.29].

Tholander, Christa (2001): Fremdarbeiter 1939-1945: Ausländische Arbeitskräfte in der Zeppelin-Stadt Friedrichshafen. Essen: Klartext. 
Trials of War Criminals Before the Nuremberg Military Tribunals Under Control Council Law No. 10. Nuremberg, October 1946 - April, 1949 (retrieved under: https://archive.org/stream/TrialsOfWar CriminalsBeforeTheNurembergMilitaryTribunalsUnderControlCouncil/Trials $\% 20 \mathrm{of} \% 20 \mathrm{war} \% 20$ criminals $\% 20$ before $\% 20$ the $\% 20$ Nuremberg $\% 20$ Military $\% 20$ Tribunals $\% 20$ under $\% 20$ Control $\% 2$ 0Council\%20law\%20no.\%2010.\%20-\%20Nuremberg\%2C\%20October\%201946-\%20April\%2C \%201949\%20Volume\%2015_djvu.txt) [Zugriff: 06.03.2019].

Wiarda, Jan-Martin (2016): Was macht die Beratung mit dem Berater? Über die Folgen von Politikberatung für die Wissenschaft am Beispiel des Max-Planck-Instituts für Bildungsforschung. Diss. Dr. phil., Humboldt-Universität zu Berlin, Dez. 2015. https://doi.org/10.18452/17433 http://edoc.hu-berlin.de/dissertationen/wiarda-jan-martin-2015-12-07/PDF/wiarda.pdf [Zugriff: 16.03. 2019].

Wildt, Michael (2002): Generation des Unbedingten: Führungskorps des Reichssicherheitshauptamtes. Hamburg: Hamburger Edition, HIS Verlag. 